This PDF is a selection from an out-of-print volume from the National Bureau of Economic Research

Volume Title: Long-Range Economic Projection

Volume Author/Editor: Conference on Research in Income and Wealth

Volume Publisher: NBER

Volume ISBN: 0-691-04141-5

Volume URL: http://www.nber.org/books/unkn54-1

Publication Date: 1954

Chapter Title: Concepts and Assumptions in Long-Term Projections of National Product

Chapter Author: Simon Kuznets

Chapter URL: http://www.nber.org/chapters/c2929

Chapter pages in book: (p. 7 - 42) 
PART I 



\title{
CONCEPTS AND ASSUMPTIONS IN LONG-TERM PROJECTIONS OF NATIONAL PRODUCT
}

\author{
S I M O K UZNETS \\ UNIVERSITY OF PENNSYLVANIA \\ A. NATURE OF PROJECTIONS
}

ALL statements about the future must necessarily employ data on past events: even the wildest fantasies are not without reference to what has been observed. The very purpose of a statement is to communicate something intelligible; and one can be intelligible only in terms of past experiences of others. In that sense, all pronouncements about the future are translations from the past.

However, in the present discussion we distinguish between statements about the future derived from tested or testable propositions concerning the past, ${ }^{1}$ and those based on theories or hypotheses that are beyond empirical description and testing. The latter, which include religious and other mystical prophecies, utopias, social and esthetic myths of various description, and plain personal hunches, are not discussed here-but not because they are unimportant. Indeed, many of them, e.g., the messianic myths of many social groups, have played strategic roles in history. We confine the discussion to statements about the future that claim to be derived from empirically tested propositions concerning the past; and we use the term projections to describe them.

Even if we have in mind only those projections concerning social or economic events, there is a wide variety of types. The broad distinction most useful for present purposes is between statements of expectations and of intentions. In the former, the observer is, as it were, outside the object to be projected; and, on the basis of past behavior, infers likely patterns for the future. In the latter, the observer is inside looking out-viewing the past as a succession of realized or unrealized intentions related to desirable goals, and considering the future in terms of goals and programs rather than in passive expectation. That statements of expectations must be based upon empirical observation of the

\footnotetext{
${ }^{1}$ For simplicity we omit references to the present. At any given moment there are only the past and the future.
} 


\section{CONCEPTS AND ASSUMPTIONS}

past is obvious. But even in statements of intentions or goals, empirical data play a crucial part. For these statements of goals, programs, plans, desirabilities-whether individual or social-are usually assumed to be feasible: otherwise they would have little sense. And feasibility can be inferred and judged only from past performance. The past, therefore, not only colors and determines the choice of what seems desirable; ${ }^{2}$ but, more important here, empirical observation of the past is essential to sound judgment of feasibility.

We are interested in both expectation and intention projections that are based upon the empirically observable past and are therefore assumed to be valid. What is the precise basis upon which the translation from the past to the future is made, and how much validity can be assigned to it?

\section{B. BASIC PREMISE}

There is a chasm between the past and the future. Empirical observation can relate to the past alone; the future is defined as the time ordinate of events not yet experienced. This does not mean that these future events are somehow there, predetermined and inexorable, awaiting only the lifting of the curtain of time to reveal themselves to helpless mankind. We do have choices within some limits set by the past. But it does mean that any projection from the past into the future cannot possess empirical validity in the way that a proposition having an identifiable referent in the past can. We cannot ask about a statement con-

2 Statements concerning desirable social ends, particularly those designated "needs" or "requirements," are governed by present, socially conditioned sets of values, thus reflecting the historical past. For example, in the goals specified in J. F. Dewhurst's America's Needs and Resources (Twentieth Century Fund, 1947), the purely biological or natural requirements of man play only a minor part. The "needs" are a rough approximation to what people want; and what they want and consider desirable is determined by how society lives, and, in a given class structure at a given level of cultural performance, by the values set by certain classes (often not at the peak of the social pyramid, not at the bottom, but somewhere near the middle). This observation is advanced not to deprecate the value of such statements: the goals are no less important because they are conditioned by the history and institutions of a given society. Indeed, it would be next to impossible to formulate "needs" in relation to physiological and biological requirements alone, and disregard the patterns of a society in supplying goods to its ultimate consumers. But such statements should not be confused with dictates of natural science-with the goals which the latter might formulate were it to define them in a tabula rasa condition-uncomplicated by a historical heritage of set social patterns. 
cerning the future, "Is it true?" as we can about one relating to some past event. All we can ask is, "Is it likely to be true?" meaning, "Are there weighty grounds for accepting it?" The answer to this question, no matter how strongly supported by elaborate empirical study of the past, is merely a judgment that cannot be fully tested; and is, in that sense, an act of faith.

The nature of this act of faith must be explicitly stated, for it involves the basic premise underlying all projections into the future. One indispensable element of the premise is the assumption of some identifiable relation between the past and the future. What is denied is indeterminacy of the relation between the past and the future. If such indeterminacy is admitted, within wide limits, if it is admitted that past events have no recognizable bearing upon the future, the very possibility of projections is denied.

But this is not sufficient, if the projection is to serve any useful purpose. Were we to view the past as a chaotic mixture of events without semblance of order, assuming that the future will be like the past could not yield useful projections since they would not limit the path of events. Nor would it help us to assume that the future, unlike the past, will show some order, since the nature of the order could not, by definition, be derived from the past. Thus projections are warranted only if the basic premise includes two elements-an identifiable relation between the future and the past, and a minimum of order in the past that can be translated into some specific pattern for the future.

The existence of order in the past is a proposition testable in the light of empirical observation and related analysis. It is the empirical foundation of this model of the past that distinguishes the projections we speak of from prophecies, utopias, myths, and hunches. It follows, therefore, that the whole possibility of projections depends on the extent to which study of the past has yielded an empirically testable picture of order in the universe. In that sense, projections were impossible before empirical science brought knowledge and understanding of the universe. The current interest in long-term projections of national product is a result of the recent development of measures covering a sufficient period and of their analysis, by which some elements of order and continuity in past changes in national product were observed. Furthermore, as our picture of an orderly universe becomes more accurate, greater control of at least some of the natural and social processes becomes possible; and this may in 
turn introduce an element of order where chaos or wide mutability existed before. Our greater knowledge of causes of death and the higher level of technical arts in general has resulted in a much more "orderly" pattern of population growth than that which characterized, say, the Western countries during the Middle Ages. The finding of order in growth of population during the last century and a half was facilitated, not only by greater knowledge, but also by better control of disturbing factors. The dependence of the orderly view of the past upon accretion of past study is thus a double one: it is affected directly by a greater knowledge of the past, and indirectly by the imposition of order by human controls based in turn upon such knowledge.

The act of faith in the basic premise is concentrated in its first element-the assumption of an identifiable relation between the past and the future. This assumption is most acceptably expressed by saying that there is no evidence that the possible future changes will exceed the limits of changing conditions under which order, i.e., some invariant or stable relations, was found in the past. Since changes in the future are hidden in that all their antecedents cannot be fully known today, the transition from the statement that there is no evidence to the contrary to the statement that there is an identifiable relation between the past and the future is a jump-which we refer to as an act of faith. But if any statement about the future is to be based upon knowledge of the past, i.e., have any empirical foundation, this assumption based upon absence of evidence to the contrary is the only one that can be used. The alternative is either not to make any statements about the future, which is a natural consequence when impending or current changes are so upsetting or so divergent from the past as to negate any bearing that it may have upon the future; or to indulge in prophecies or hunches, also a rather common practice when no apparent order can be found in the past or when conditions bear a strongly apocalyptic tinge.

\section{MAJOR PROBLEMS IN ECONOMIC PROJECTIONS}

The finding of some orderly pattern in the past and the assumption of a determinable relation between the past and the future encounter major difficulties in economic projections, particularly those expressible in quantities. These difficulties, in connection with long-term projections of national product, are discussed in 
general terms in Sections $\mathrm{C}$ and $\mathrm{D}$ of this paper and the more specific questions relating to the structure of the totals, character of the levels, and time extension are treated in later sections.

As already indicated, establishing some order for the past depends upon the extent to which accumulation and analysis of data have revealed some persistent pattern. The major difficulty in finding such patterns for national product arises partly from the scarcity of data, and partly from the limitations of past analysis. The limitations will be clearly perceived if we assume what actually is not so: that we have a tested theory of the economic growth of nations which demonstrates that long-term changes in national product invariably follow a specific pattern expressible by a given curve; that this pattern is securely founded upon a causal explanation that traces it to underlying factors that in turn display persistent patterns of change-say, trends as clear-cut and as invariant as in the growth processes of a biological species. With such a theory, the estimation of specific constants for any given nation for any given period would still raise questions ordinarily involved in fitting a theoretical model to a body of empirical observations. But these questions would be minor compared with the difficulties, and the pitfalls, that loom in the absence of such a theory, or when the study of the past has yielded hypotheses so vague that an uncomfortably wide choice of systematic patterns of long-term change to describe the past is possible.

So far as I know, we have no adequate theory of economic growth of nations, and none that would securely establish a specific model of long-term change in national product. Without it, attempts to find such patterns are based upon direct observation of specific series relating to national product (or its immediately determining components, such as population and per capita product), and result in models chosen on the basis of such observation alone, without explicit reference to the varieties of experience that would be subsumed in a tested general theory.

Under such conditions, the pitfalls are numerous indeed, and, despite our cognizance of them, cannot easily be avoided. To illustrate: When we state that the per capita product in the past increased, on the average, $x$ percent per decade and use this observation as a basis for projection, we imply that this particular pattern of change, this increase of $x$ percent per decade is a reflection of some force, some element of invariancy that persisted in the past despite changing historical conditions. But if this 


\section{CONCEPTS AND ASSUMPTIONS}

$x$ percent is a simple average for $n$ decades, there is no assurance that it represents a persistent pattern. Were we to divide the decades into two groups, the earlier one might show an average increase of $x+a$ percent per decade, and the later of $x-a$ percent. And if we try to handle this complication by simple curves that allow for a systematic acceleration or deceleration of the rate of increase, we find that a great variety of curves can provide a fairly adequate description-each, however, yielding different projection levels for the future.

Since these problems are familiar to anyone who has experimented with fitting lines of secular movements to economic time series, guided by purely empirical criteria of goodness of fit, there is no need for elaborate discussion. The crux of the difficulty in establishing an orderly pattern of long-term change lies in the fact that, in the absence of effective theory or even of working hypotheses, a great variety and wealth of data are needed to discriminate among the many models that can be used to describe the major characteristics of change. Yet no such variety of data is available, within the limits of societies reasonably similar to ours. With the available data, it is extremely difficult to choose even among the simple models used to describe the underlying, primary secular trends. Yet our projections into the future will differ significantly as we use one model rather than another. This can be demonstrated by fitting three curves-the logarithmic parabola, the simple logistic, and the simple Gompertz-to, say, estimates of real national product in this country, either total or per capita, for the period 1870-1940, and then extrapolating to 1980 . If, in addition, we cover under long-term changes secondary secular movements, trend cycles, or long cycles, whichever term one wishes to use, the possibility of deriving a given pattern becomes even more remote. The power of discrimination which our limited data permit us to exercise in choosing among the possible patterns for purposes of projection is still weaker.

But let us assume that this major difficulty of selecting the proper pattern of systematic change to describe the past has been solved. We still face the next problem: Can this pattern be projected into the future?

This problem must be treated in the light of the following considerations. First, the future will necessarily contain some elements that did not exist in the past, if merely because it comes later on the scale of history. Indeed, at any given time, there are 


\section{CONCEPTS AND ASSUMPTIONS}

indications or portents of impending changes, and taking an inventory of them for purposes of appraisal is one way of dealing with this translation from the past to the future. But our knowledge of such portents, no matter how rich, is never adequate; and particularly it must be evaluated to ascertain whether these "new" changes are similar to those that accompanied or entered into the pattern of past change which we are ready to project, or are so entirely new as to belong outside the range of past changes.

This introduces the second consideration, that of the range of changing circumstances under which the persistent pattern of long-term movement was found for the past. If we establish empirically a long-term trend for a period during which great changes in possibly relevant conditions occurred, this pattern has more significance than one established for a period during which the potentially relevant conditions changed but little. For unless it can be assumed that the future will be relatively free of changes in relevant conditions, projecting into it a pattern whose persistence has not been tested by a diversity of historical conditions is a risky step.

Whether the past can be projected into the future thus hinges upon whether possible future changes, insofar as they can be foreshadowed at present, can be compared with the range of relevant changes in the past within which the systematic pattern persisted. Such a comparison requires: (1) knowledge of the factors and conditions that are relevant to the object of the projection, i.e., that can exercise significant effect upon it; (2) ability to appraise the magnitude of impending changes in relevant factors; and (3) ability to appraise the magnitude of past changes in relevant factors.

Little can be said about the first aspect, except to stress that at this point also the absence of a well-founded theory is crucial. Such a theory not only establishes the connection between the dependent variable and some independent variables and yields some general model of the pattern of change in the former over time; but also provides a relatively complete system, which permits us to treat everything outside it as irrelevant. For example, if the theory held that the basic and only factors in long-term changes of national product were growth in technology and in the number of workers (with these in turn reducible to, say, hereditary genius phenomena governed by biological law and 
by invariant tendencies to human reproduction), then we could ignore political changes and revolutions, institutional habits, international disagreements, exhaustion of natural resources, and the thousand and one items included under these broad heads. But we have no such theory, and hence the field of our vision is distressingly wide and the possibly relevant factors numerous. When we attempt to find the past variations in relevant factors that accompanied the persistent pattern established in growth of national product, we flounder in a motley variety of historical events. Likewise, when we ask whether the changes in the future, whose antecedent signs we see, or think we see, today, are within the range of changes that characterized the past and yet yielded a persistent pattern, the answer can only be a rough judgment. ${ }^{\mathrm{s}}$

Thus two major problems in economic projections are establishing a systematic pattern of past change and gauging the variety of relevant conditions under which this pattern was found

${ }^{3}$ It is perhaps dangerous to look at the greener pastures of the experimental scientists and ask how they do it-dangerous because in our ignorance we may misinterpret and overaccentuate the difference. But when a change can be deliberately imposed for the purpose of studying its effects, the situation is different from that for economic projections. The value of the controlled experiment lies in the power of the observer to isolate the object of study from the variety of circumstances that might affect it, and in such isolation to distinguish the major determining factors and exclude the irrelevant ones. It is immediately possible to discriminate finely among alternative theoretical models, and to specify the conditions under which the model selected remains invariant. If these specified conditions can be reproduced, as they ordinarily can at least en gros, and if the object of study has an empirical referent, the controlled experiment results in an admissible projection into the future to the extent that if certain conditions are fulfilled (and they can be at will), recognizable results will follow either invariably or with a tolerable margin of variance. For example, if certain recognizable material elements are heated under specific reproducible conditions, and the pressure on the walls of the vessel is measured, this pressure will follow an invariant pattern. These invariant conditions can be reproduced and this model has a recognizable counterpart in reality. In the social sciences, where controlled experiment is impossible, one can simulate controlled conditions by the use of the imagination. But then the object of study loses its empirically identifiable character and becomes a construct for which there is no direct counterpart in reality. The conclusions established in this manner could be "projected," but they would not be useful because they would bear no determinate relation to the real worldessentially because the conditions cannot be reproduced as they can in a genuine controlled experiment. For example, we can deduce the behavior of "economic man" in a given market situation, under static conditions; and we can project it into the future by assuming the persistence in time of both "economic rationality" and static conditions. But this would hardly be useful because the imaginary conditions thus set cannot actually be reproduced. 
to persist. The former difficulty is fairly familiar, and the various technical means for dealing with it, as well as the pitfalls to be avoided, are reasonably well known. The latter is less amenable to technical solution, and is in practice resolved by a whole variety of assumptions. These assumptions are crucial and deserve separate discussion.

\section{TYPES OF ASSUMPTION}

Any economic projection, particularly an inclusive one like national product, carries with it a host of assumptions-so great and varied that any attempt to group and describe them will probably be incomplete. The summary below bears this unavoidable qualification.

1. The first group of assumptions concerns continuity of physical nature: that the laws that govern the physical universe, as observed by empirical science in the past, will continue-whether they relate to inanimate nature, biological species, or the psyche of man. Since all human activity rests upon a physical foundation, such an assumption is indispensable in any social projection. It is never stated explicitly in presenting, say, a projection of national product because it is taken for granted. But this assumption, which is borrowed from sciences relating to factors exogenous to human society as such, is still an assumption-no matter how deeply ingrained our belief in it is. Furthermore, economic and other social projections would be impossible without the antecedent development of our understanding of the processes of physical nature; e.g., in the intellectual climate that existed prior to the development of empirical science and when there either was no assumption of order and continuity in the physical universe; or when such order was attributed to some mystical force that could change in unknown ways and whose ungovernable changes could radically modify the physical basis of social life.

2. Another type of borrowed assumption relates to factors or forces that may be outside and not affected by the social system but which may affect it much more specifically than the borrowed assumptions under (1). The expectation that physical nature will operate as it did in the past permits us to state that society will continue to function on the same broad physical foundation, but it does not limit or specify any particular magnitudes for the 


\section{CONCEPTS AND ASSUMPTIONS}

performance of human society. With all the laws of physics operating, national product can go up and down by very wide margins. But there may be data about the physical universe, truly exogenous to social phenomena, that can determine future social magnitudes in a rather specific way. We might call these the "specific borrowed projections," which trail a whole host of assumptions behind them. For example, an assumption derived from technological study might be that rise in efficiency of power use represented by BTU output per unit of input of a given mineral follows a given specific curve. A still better example would be the as yet nonexistent science of technological change that might supply economists with specific assumptions about the pattern of future technological change, which could then be used directly as independent variables to derive specific national product projections.

These borrowed assumptions, or borrowed specific projections of determining factors outside the economy may relate to factors that are not truly exogenous, but are in fact partly determined by social processes. Consider the population projections borrowed by economists from demographers. These projections are accepted on the ground that demographers use all available information on past patterns of births, deaths, and migration, and are better judges than economists of the specific assumptions that can most reasonably be made. That much is true; yet it is also true that population growth is not truly exogenous but is dependent upon economic and social conditions. Hence, these specific projections mean borrowing also some specific assumptions concerning social and economic factors, and these borrowed assumptions may well conflict with those the economist explicitly makes when he develops his own premises. For example, it is not clear to what extent recent and current estimates of population growth are based on the assumption of full employment and of all that it portends for the birth rate, family formation, etc. Nor is it clear what the population forecasts assume about peace or war. And what is true of projections for population may well be true of borrowed specific projections for technological change, state of irreproducible natural resources, and the like. Indeed, the exogenous character of any specific projection should be suspect when it is borrowed by an economist, because it may bear directly upon the specific magnitudes of his projection of national product-unless it can be proven otherwise. 
3. The third group might be designated "general assumptions concerning social continuity." Because such continuity is somewhat more doubtful than that of the physical universe, these assumptions are usually explicitly stated, e.g., a projection is made on the assumption that the United States economy will remain one of private enterprise, business competition, democratic government, etc. Such statements, however, do not bar all changes: they presumably permit some modifications compatible with the continuity of broad bases of social and economic organization. The difficulty is to know which changes do and which do not violate this assumption of continuity. In national product projections accompanied by statements using the language just employed, would a continued extension of large-scale enterprise to the point of reducing effective competition in most industries be considered a break in continuity? Would nationalization of a few basic industries, say, coal mines and the iron and steel group, constitute such a break? Or formation of overpoweringly strong trade unions? Or, for that matter, further development of the recent practice of using resources for foreign aid without an economic quid pro quo?

It is easy to be overimpressed by current or impending changes, and to view the past as sharply severed from the future. Yet even a glance at the succession of past changes that seemed revolutionary to many contemporaries, and are not unimportant even in retrospect, shows that many systematic patterns established for the past persisted amid quite a turbulent stream of historical events. When the land frontier was closed, when the trust movement began, when the income tax was introduced, when free immigration was suspended-there were always contemporaries who viewed the change as the end of an era. Yet certain basic patterns of change in the country's national product and its major components persisted through and after these changes. There is, therefore, a sound instinct, if only an instinct, for assuming a general continuity of social behavior-except when the evidence of catastrophic or extraordinary change is overpowering.

Since the assumption of continuity is essentially a judgment to the effect that possible changes in the future, foreshadowed at present, are within the range observed in the past, the observer usually resolves his doubts by attaching limiting conditions. For example, a statement may be attached indicating that the projection is based upon the assumption that the relatively peaceful 


\section{CONCEPTS AND ASSUMPTIONS}

conditions of the past will persist, and that the occurrence of a war will invalidate the projection. By doing this the author of the projection appears to transfer the responsibility to the userhaving warned him in advance of the possible contingency that clashes with the basic assumption used. But is such a shift of intellectual responsibility genuinely possible? The very raison d'être for the projection, and its publication, is to indicate acceptable possibilities. Otherwise, one could always convert the projection into an irrefutable tautology by attaching a statement that it is valid "if the future changes are like those in the past." But such tautology is completely useless, since strictly speaking the future can never change in exactly the same way as the past. If anything more than a tautology is involved, we must express a judgment as to reasonable probabilities and bear the responsibility for it. Any specific condition that is attached, e.g., that of "no war," is usually made explicit precisely because it is a possibility; otherwise there would be no reason to formulate that specific limiting condition rather than another. A projection with such a limiting condition is justified only if, in the author's judgment, the probability of the limiting condition is significantly lower than that of the assumption of continuity. ${ }^{4}$

4. The last category of assumptions may be designated "specifying conditions." They specify in advance either the magnitude of some components of future national product, or some goals which the projection must satisfy. The reasons for making such assumptions vary. In some cases they are made because intentions of certain economic agents, e.g., of specific government programs, are known, and these intentions are expected to be carried out. In other cases, we may have a program aiming at a general goal, viewed as a socially overriding objective, e.g., full employment; and satisfaction of this goal becomes a specifying assumption to which the projection is geared. In still other cases, certain future contingencies, not necessarily revealed in the past, may be considered highly probable, regardless of their desirability; and they are therefore specified to assure that sufficient attention will be given to them in arriving at the projections.

When these specifying conditions or assumptions are within the range limits of past changes, no particular difficulty is met in using them. For example, if future government programs are

4 See Harold F. Dorn's "Pitfalls in Population Forecasts and Projections," Journal of the American Statistical Association, September 1950, pp. 311-34. 


\section{CONCEPTS AND ASSUMPTIONS}

known, and are close in magnitude and type to those observed in the past, their inclusion in a specifying assumption can only help in making a projection-since concrete values are provided for some components, without disturbing the persistent relations within the system of components that prevailed in the past and that can be extrapolated into the future. But in other cases, the specifying condition is explicitly formulated precisely because it represents a change from the past-something new that either has not been observed before, or has been observed under circumstances that do not permit an easy translation into the future. Such specifying assumptions may well be in conflict with the basic assumption of social continuity under (3); and their acceptance and proper application involve grave intellectual risks.

Consider two specific illustrations to which we have already alluded. The first is the assumption of full employment. Even if we succeed in defining this condition precisely, say, in terms of maximum frictional unemployment allowable for given secular levels of national product, how can we use the data for the observable past when full employment was absent-except in highly transient phases of business cycles which by that very fact did not provide a reliable picture of secular levels and relations? In trying to fit this new element into the picture, we must revise the version of the past, and somehow find analogues to secular conditions under full employment. Similarly, in the second illustration, that of the unfortunately necessary current assumption of continued cold war or hot peace, the specifying condition cannot be observed in our past. It is certainly not found in years of peace, and the experience of our all-out wars is not a proper parallel, since it is for short-term efforts rather than long-term ones. Any attempt to incorporate such a specifying assumption must, therefore, adjust the picture of the observable past in some imaginative way to provide a reasonably acceptable analogue.

This subcategory of specifying assumptions referring to something really "new" is in a different class from the three other types of assumption described above, or from the specifying assumptions that clearly stay within the limits of the past. All the other assumptions stress continuity; the present subcategory specifies discontinuity. Assumptions in this group introduce large elements of judgment and uncertainty into the projection.

No general account of the solution to this problem can be given: it constitutes a substantial part of the several papers that 
deal with the various components or aspects of national product projections. But we point out that the problem exists because it is assumed that large sections of the economy will continue to operate as free agents; and it is their reactions that are difficult to gauge under conditions so different from the observable past. If, however, we extend the specifying conditions or assumptions to cover by fiat the whole economy, the problem disappears, or is at least modified. It ceases to be a matter of the probability or cogency of a projection, and becomes essentially one of technical feasibility of an over-all plan.

To state the character of this shift more explicitly: the specification of an over-all plan as a condition implies the full exercise of coercive power by a necessarily authoritarian state-even if the decisions of the latter may follow some discussion of desirabilities. We assume here an established authoritarian state, past the turmoil and stress of the period of its coming into being-without relevance to any current or possible experience in this country. Once the over-all plan has been decided upon, the question is one of technical feasibility-of availability of physical resources, including man. The decision upon an over-all plan implies disregard of the economic and social values of individuals as free agents. Economic attainment may cease to be a question if the paramount objective is to be reached regardless of sacrifice and costs. It is in this sense that the problem becomes one of technical feasibility, rather than of economic probability. ${ }^{5}$ This perhaps overdrawn picture is useful here because it points up the observation that the problem of feasibility involved in national product projections lies in the reconciliation of certain specifying assumptions that represent overriding intentions or programs of a type unknown in the past with such free behavior in the major sectors of the economy as represents continuity with our observable past. Obviously the problem can be resolved only tentatively and with a wide margin of error. Also, its effective handling requires a clear line of demarcation between the overriding intention and the free sectors of the economy; a limit at which this intention

5 This has been the experience with over-all economic plans whenever introduced. That short-term economic objectives are assured under such conditions of pressure by an authoritarian state does not preclude a distinct possibility that the system may prove far less efficient in the long run than a social organization in which less centralized planning and a much greater scope for individual decisions by free economic agents prevail. 
stops by design or must be curbed by the area assumed for the free economic activity.

\section{E. STRUCTURE OF THE TOTALS}

In projecting national product, we must obviously go beyond over-all totals and differentiate major components. National product measures a cross-section of a continuous stream of economic activity, of a flow of resources into production, and of the outflow of finished products which in turn yields new resources and further production. A proper structuring of the totals-differentiation of components that are direct antecedents, contents, and effects of national product-is indispensable, no matter what uses the projection may serve. Even if the over-all totals can be derived without breakdown into components, the latter are needed for a more careful check on the reliability of the projection in the light of past experience-a check in terms not only of global indexes for the whole economy, but of the projected performance of various sectors which stand for institutionally distinct groups in society whose habit patterns may be sufficiently known to permit judgment as to the acceptability of the projected levels. If, for example, the over-all projection implies magnitudes of food output for domestic consumption that yield an unreasonably high per capita figure, the total itself may be questioned. If specifying conditions or assumptions are introduced that set the magnitudes of some components in advance, then, even though an overall total may be derived directly, the other components must be approximated to see whether their relation to the assumed components violates expectations based on past behavior. In other words, the very technique by which national product projections are derived and tested requires that as many components be distinguished as there are sectors in the economy characterized by different behavior patterns. Over and above this need, such breakdowns are indispensable in any use of projections for analysis or for policy action, since the latter should be geared to separate sectors of the economy-not necessarily to the nation's economy as a whole.

In practice, many current projections of national product are first calculated as over-all totals, usually by multiplying labor force (derived in turn from specific projections concerning population growth) by per worker productivity. Then, on various 
bases, the several components of national product at different stages of circulation are projected (at the level of production, distribution of income shares, consumption, and investment). Next, these components are tested for consistency with each other (whenever determinate relations exist), consistency with the past, and finally consistency with the over-all total first derived. At the next round, modifications are made in components and/or in the over-all totals to attain a consistent set of estimates of both the totals and the various components. This oversimplified description omits several rounds of revision and reconciliation, some of which may involve substantial adjustments of steps that yielded inconsistent or otherwise unacceptable results. But it indicates, at least, the reciprocal checking of the over-all totals against the parts, and of the parts against each other.

We are interested here only in how this breakdown of the totals affects the reliability of the projection-not its uses for analytical or policy purposes. And if we ask how such a subdivision yields a more acceptable projection than one limited to a single over-all total derived in the customary way (or any other manner, except that proceeding by parts), the answer must be sought in the light of the following considerations.

First, it may be stated, on purely empirical evidence, that the over-all totals show a greater inertia, a much simpler discernible pattern of movement over time, than most of the components usually distinguished. This observation is clearly illustrated in a comparison of the secular movements of total population in the United States and in a much narrower area within it, say, a single state. Clearly, the trend lines in the former display a simpler and steadier pattern, and their changes over time seem much more limited. The same conclusion would be reached if we compared long-term changes in total national product with those in product originating in any industrial sector, any geographical region, the shares represented by any income type, and the like. This does not mean that it would be difficult to find some components whose secular trends move as slowly and apparently as evenly as those of the over-all totals (the discussion throughout is in "real" terms, free from the disturbing mobility of price level). But it does seem true that the over-all totals display more stable patterns of change over time than most components.

The theoretical explanation of this empirical finding is beyond my power to provide. The direct reason can be clearly seen. Total 
national population is blocked off by legislation and other barriers from violent changes by immigration and is moved largely by rather inexorable factors underlying birth and death rates; whereas in the case of population for a single state, internal migration, a more volatile process, may be of paramount importance. Likewise, given the basic trends in total population, and deeply set institutions determining labor force participation, consumer habits, and the like, we have a basis for expecting stable trends in total output, unless major disturbing conditions are assumed; whereas in any single industry, the possible impact of technical change and of change in taste may produce violent fluctuations over short periods. But these are superficial observations; the underlying mechanism that explains this relative stability of over-all totals combined with the competing and offsetting and more rapid changes in the components is far from clear. ${ }^{\circ}$

Second, and in contrast, it is easier to understand both the trends and the limits of the narrower areas of observation-the components-than those of the over-all totals, whose composition is so variegated and complex. If the present domestic consumption of prunes per capita is 2 pounds a year, and has varied within a range of, say, 1.5 to 2.5 pounds over the last fifty years, a

6 Cancellation of random changes, which makes for greater stability (narrower sampling errors) in measures treated in statistical theory, is hardly relevant here. In the changes in components, fluctuations that can be attributed to random factors are among the least important. It is rather that when we deal with a social aggregate, certain patterns, traceable perhaps to some basic characteristics of human nature or to deeply ingrained institutional habits, emerge and are impressed on the resulting totals. Thus it is generally true that for society as a whole, an increase in per capita income is accompanied by a decline in the proportion of income spent on food; the same two variables are differently associated when the several subgroups of food or of society are considered. It is generally true that in societies permitting consumers some freedom (and even in some authoritarian societies), the secular level of the savings-income ratio has a fairly low upper limit; this is not true for some subgroups in the society. Both results may be traced to the nature of human wants and to the general habits of the human race in apportioning its resources and attention between the present and the longer future. And yet it may be dangerous to generalize about these patterns of behavior of social aggregates, except within some limits set upon characteristics of social organization. One might ask, thinking of the Lucullan feasts, whether in Roman society an increase in per capita income over time was accompanied by a decline in the proportion of expenditures on food; and whether in a well-established authoritarian society like Egypt in its ancient heyday, the upper limit of the secular savings-income ratio was confined to the $15-20$ percent range that seems to have been typical of Western societies of the nineteenth and twentieth centuries. 
projection of per capita consumption to a date fifty years from now that yields a value of 10 pounds per capita can be treated as suspect-because we know what prunes are and can limit the possibilities involved. But if we substitute for prunes total consumer expenditures per capita or total product per worker, we can no longer be intuitively knowledgeable because these entities include so much. It is, therefore, difficult to see why the possible changes in them should be limited. ${ }^{?}$

In the light of these considerations, how can the distinction of components serve as a check upon the projected national product totals? It cannot provide a check directly: an over-all total derived by summation of projections for parts is not necessarily more acceptable than one derived on a global basis. The check can be provided on only one of two assumptions. The first is that some of the specific components are of strategic significance as determinants of total national product levels; and that a check on their magnitudes in the light of what we can tell about their reasonable limits is possible. This is the essence of all national product projections guided by the Keynesian stress on the strategic role of capital investment as an offset to savings. Acceptance of the theory, in its full implications of the possibility of secular disparity between ex ante savings and possible outlets for capital investment, would compel an author of a projection, after deriving an over-all total, to check whether the implicit volumes of business capital formation needed to offset the implicit savings are at all likely, in the light of what we know from the past about business capital formation and savings habits. The difficulty with this and similar theories, in their use along the lines suggested, is that we know relatively little about past behavior of such processes as flow of savings or business capital formation. It is, therefore, difficult to assign relatively accurate values to their

7 The easier understanding of trends in narrower components should not be taken to be, in and of itself, a safe basis for setting limits to their possible changes in the future. If it is suddenly discovered that prunes contain the elixir of youth, a rise in consumption to 100 pounds per capita is not out of the question. However, for these narrower areas, more specific information may be at hand to provide a basis for intelligent judgment concerning the limits of future changes. This does not deny that for the larger aggregates the empirically observed basis for assuming relative stability in secular movement is much wider; and that study of these wholes, rather than the parts, may reveal some effects of social processes that cannot otherwise be clearly discerned. That the whole is greater than the sum of its parts is clearly reflected in the common reference to the economic or social "system"; by definition, "system" is much more than a sum of parts. 
projections, at any rate as compared with the greater relative accuracy of the over-all totals themselves, and particularly to pass judgment on their acceptability. I would, therefore, be inclined to argue that this way of using the strategic components to check on the plausibility of over-all totals is a weak reed.

There is another way provided by a different assumption. The past growth of national product was accompanied by a fair amount of internal shifting of components. As already noted, the relatively stable trends in the over-all totals are due to the combination of more rapidly moving, diverse trends of components. The projection of any over-all total into the future, yielding a certain secular rate of change, implies some further shifting in relative weights of various components. The breakdown of the total may reveal how much shifting is implied in the projected change of the over-all total; and comparison of the extent and character of the shifts so implied with those observed in the past can shed some light on the acceptability of the projection. Assume for the sake of illustration that a projected total implies a shift out of agriculture involving a reduction of the farm population to onehalf its current size in 20 years, or a shift of labor force from the East to the West of proportions not hitherto observed, or that are extremely unlikely with the much smaller mobility of population than existed in the nineteenth century. The question is whether we can use the breakdown to compare the rapidity of the implied shifts of resources or of changes in economic behavior patterns with those observed in the past and considered likely from present observations. ${ }^{8}$

Our knowledge of the past may not be sufficient to permit this type of testing of over-all projections. But as our knowledge of relations among components increases, as our understanding of the factors that determine the mobility of resources in their shift from one sector of the economy to another grows, and as our information concerning behavior patterns is more thoroughly analyzed, the value of subdivision of totals into significant components for checking the whole projection, even the over-all totals, will become greater. But even at present, it may be well

8 Under conditions of authoritarian compulsion, this problem of mobility of resources and changes in behavior patterns is greatly reduced, although not completely removed. In such states, mobility and change in behavior patterns are subject to forceful manipulation. This is much less true in nonauthoritarian states, although the latter may use stimuli that are not much less efficient in the longer run. 
to pay more attention to the past movements of the components as indicators of the rapidity with which shifts of resources, or changes in patterns, can occur. They may be useful not only in testing the over-all totals, but also in assigning some margins of error to the component parts of the projections themselves.

In this connection, a comment might be made concerning one assumption ordinarily attached to national product projections that is ambiguous in meaning. Projections usually refer to national product totals and components in a given year's fixed prices. The ambiguity is revealed when one considers that the projection must involve changes in relative weights of components over time. Does the assumption mean that not only the general price level, but also the relative prices of goods in various sectors of the economy, are fixed? Does it mean that the price ratios of various groups of goods remain as they were in the year that marks the date of the assumed price level? If it does, then one major element that permits shifts in the relative importance of various sectors and that presumably helped to bring about such shifts in the past is forcibly removed by the assumption; and this removal jeopardizes the economy's ability to adjust to the shifts implicit in the projected secular changes in the national total. If the assumption of a constant general price level still permits shifts in relative price ratios, should not such shifts be explicitly introduced in the formulation and calculation of the components of the projections? In this case, the projections for the components must be shown in both the prices of the given year (current) and those of the year that dates the projection.

In this whole area of structure of projected national product totals, past shifts must be studied from the standpoint of capacity of the economy for making the adjustments involved in any overall rate of growth, and the ambiguity involved at present in the assumption of constant prices must be cleared up. One concluding observation may be made. When a national product projection is fully presented with both over-all totals and a variety of significant components, the errors attaching to at least some, if not most, of these components are relatively greater than those attaching to the broader totals. We recognize that it is not easy to specify the margins of these errors. But at least some indication of the difference between the margins of error for the totals and for the components should be given, and the range of pos- 
sible variation in the distribution of the projected over-all totals among various possible combinations of components indicated.

\section{F. CHARACTER OF LEVELS}

When national product (or any other) projections are described as "long-term," the levels are assumed to be descriptive of the longer run, rather than of any short period. Even if the projection is labeled for some single year in the future, it is still implied that the secular level is being approximated, not the level for a single year that would reflect the phase of the business cycle or other transient phenomena. The same implication is often expressed in the term "sustainable" applied to a projection: sustainable means presumably a level which, with allowance for limited short-term fluctuations about it, will describe a long period -not just a level barely attained in a short-term shift, to be succeeded by persistently lower ones.

Furthermore, this long-term character of the levels is assumed to apply not only to the over-all totals, but also to the significant components: it is not only the national product level, but the levels of the various components and the relations among them that are assumed to be sustainable. Any ambiguity or difficulty attaching to this characterization of levels will, therefore, apply both to the over-all totals and to the significant components.

This ambiguity resides in the relative, rather than absolute, character of the concept of long-term, secular, or sustainable levels. Secular levels, usually defined as movements in one direction as contrasted with cycles or other shorter-term changes which involve a frequent reversal in the direction of movement, are a relative concept. On a scale of centuries, many of what we now call secular movements in time series would be cycles. Contrariwise, we may, on the kind of time scale with which we operate, distinguish primary and secondary secular movements, underlying trends and trend-cycles. What level are we considering in long-term projections of national product? For how long a period are we concerned with sustainability?

The problem may seem to be easily resolved by defining sustainable levels as those that are maintained without any longterm absolute decline, specifying the "long term" as a period of over three years. For example, a projection of national product of $X$ billion dollars for 1980 is meant to be a long-term level for 
that year, not one caused by a cyclical boom condition, and we seem to remove ambiguity by saying that no negative departure from that level lasting more than three years (or two or one) is expected after 1980. This answer may appear to free us from the need of worrying whether by a secular level for 1980 we mean a level on the primary trend line sustainable over periods associated with recognition of long cycles, or a level on the line that includes both the underlying trend and the long trend-cycle (or secondary secular movements).

But there are two difficulties with this easy solution. The first is suggested by the following illustration: Assume that the condition set, no absolute decline after 1980 , is fulfilled, but that the percentage rates of increases in 1981, 1982, and so on are barely perceptible, whereas before 1980 the projection showed substantial rates of increase. Would the level for 1980 still be considered sustainable? Does not sustainability refer to the level as an item in a systematic long-term change, i.e., to the rate of change over time, and not merely to the absolute level? If so, the possibility of variations in the rate of secular growth itself introduces some ambiguity. Should we allow sor such short-term variations in the rate of secular growth, in the rate of change in the long-term levels predicted by our projections? And if so, within what limits?

The second difficulty with the easy answer concerning nonreversibility of absolute changes as definition of sustainability lies in the components. If we accept variations in the rate of secular change in over-all totals as compatible with the criterion of sustainability, if we say that the projection represents a sustainable

${ }^{9}$ An illustration of this difficulty is provided by calculations recently made at the National Bureau in the study of capital formation and financing in the American economy. In this calculation, nine-year moving averages of national product and its components were computed from annual estimates based largely on data for overlapping decades originally published in National Product since 1869 by Simon Kuznets (National Bureau of Economic Research, 1946). These moving averages remove almost all the shortterm fluctuations associated with business cycles. They reveal rather marked longer-term swings in the rate of growth. Thus from 1873 to 1883 (dates of midyears of the moving averages) the rate of increase per decade in gross national product ( 1929 prices) is 91.0 percent; from 1883 to 1892 it is 37.3 percent; from 1892 to 1905 it is 53.4 percent; from 1905 to 1911 , 30.6 percent; from 1911 to $1926,39.0$ percent. In no case does the absolute level of the moving averages decline; the swings are in the percentage rate of growth. Should projections of sustainable secular levels correspond to these variable rates of growth or to the primary trend lines underlying them? If the latter, how much variation in the rate of growth around that line should we permit? 


\section{CONCEPTS AND ASSUMPTIONS}

level, even if the subsequent rise in total national product is limited to one-thousandth of a percent per decade (as compared with 30 percent prior to it), we must also allow for the nonsustainability of some components, since they, unlike the total, would show absolute declines. This follows from the observation, for which there is substantial empirical evidence, that the shorterterm variations in the rate of secular change are much more prominent in some components of the national product (e.g., in construction, particularly residential, and in investment in transportation) than in others. Will it violate our criterion of sustainability if the projection permits, shortly after establishment of the levels assumed in it, substantial absolute declines in some important components of real national product?

I am raising these questions not because I have an answer to them, but because we should be aware of them, and because the meaning of sustainable long-term projections needs clarification. The ambiguity resides partly in possible differences in the meaning of cycles whose removal is identified with the long-term levels, i.e., with sustainability; and partly in the question whether such sustainability, however defined, is to be applied to the over-all total alone or to its major components also.10

However these questions are answered, accumulated experience and analysis from the past are required to distinguish between secular levels and shorter-term fluctuations associated with business cycles, and between various types of long-term movements within secular levels. It is in this connection that the scarcity of data for the past is particularly limiting, since these problems require information and analysis for a fairly long stretch of experience. If we have a theory, or at least a set of working assumptions, that permits us to consider social processes in the United States in, say, 1870 and 1950 as comparable, then we should have and use data for the full period to discern various types of secular movements and distinguish them from business cycles. It was the lack of such data that led in some recent projections to an attempt to use the short stretch from 1929 onward as a basis for long-term extrapolations. Yet the attempt to derive from what are essentially short-term adjustments some bases for

10 The same question applies to the definition of full employment, which is a closely related concept. Does full employment allow for frictional unemployment for the longer range, unemployment that may be associated not with the shorter cycles in general economic conditions, but with the 18-year cycles in residential construction? 
estimating the longer-term movements of the economy is particularly likely to lead to fallacious results.

One other point is relevant here. Projections into the future are ordinarily built up from the current year. But one cannot start with the data for the most recent year and assume that they are at a secular, sustainable level. The current year may well be above or below the trend line, however one defines the latter. At any given time we are at some phase of the cycle, not necessarily where it crosses the trend line (even if we disregard other transient disturbances not included in the concept of business or economic cycles). True, in some procedures, attention has been paid to this point and some magnitudes and relations for the current year have been disregarded or adjusted on the assumption that they have been affected by transient circumstances. But such an adjustment is not an easy matter. We are never sure in what phase of the cycle we are, until the cycle has been completed; and we are never sure that a given cycle is completed until the next cycle has definitely begun. In other words, how a given year relates to the secular level, to the sustainable trend line, can be determined only when the year is safely past, and when enough time has intervened to define the pattern of shorter-term changes. Yet in projecting forward, one is always tempted to start with the very latest date for which information is available, since thereby one extends the period under study and secures data for the most recent performance of the economy upon which to build the picture for the future. Consequently a conflict exists between the need for an assuredly secular, sustainable level as a springboard for a projection of secular, sustainable levels in the future and the desire to start with the most recent levels which the economy has attained. How this conflict is resolved can best be discussed in specific terms. But some cognizance of it helps us to make a proper choice and, particularly, reminds us that longterm projections should not be automatically based on the latest year for which figures are available.

\section{G. TIME EXTENSION}

The comments just made concerning sustainability of levels in long-term projections have a direct bearing upon their time extension, i.e., the period of time ahead which they have in view. When a projection is calculated for 1980 , it would seem at first 
that the reference is to a point 30 years from today, and not beyond. But when we say that the levels are sustainable, it means that they lie on some line of secular movement-which implies that they are part of a series of changes that will not reverse its direction shortly thereafter. A projection of sustainable or secular levels for 1980 is by definition a projection beyond this datehow far beyond depends upon the character of the secular trend implied. One must therefore envisage a long-term projection into the future as having a time extension significantly beyond its specified date, only gradually fading away into the more distant horizons of time.

While this observation may at first seem a formalistic fancy, it has an important kernel that should be made explicit. This can be done by an extreme example, similar to that cited in the preceding section. Assume that in the projection for 1980, which we characterized as one at sustainable levels, we accepted specific conditions or assumptions (say, some investment-inducing legislation) that would favor the attainment of these levels by 1980 but would cause an extraordinary pressure on reserves and resources of the economy which would not necessarily be reflected in any customary depreciation. measures. If analysis were to show that the specifying assumptions of the projection were as described, and if it were also to show that there is little ground for doubting the attainability, under these specific assumptions, of the national product levels posited for 1980 , would we still be willing to describe the projection for 1980 as sustainable? And if we hesitate to do so, is it not because we foresee that after 1980 the past rate of secular increase would be jeopardized by the means taken to achieve the 1980 levels? If so, it is clear that when a given national product projection is said to describe sustainable levels for 1980 , the implicit time extension of that statement reaches well beyond 1980 .

This basic consideration gives rise to other, related questions. The first concerns the limit beyond which the time extension of any projection cannot go. There is obviously no infinite series by which a projection of sustainable levels for 1980 implies some vaguer projection for the year 2010, and by which the latter implies another projection concerning the year 2040, and so on. Social phenomena do not lend themselves to such mathematical play. This kind of time series is quite sharply terminated by the realization of our ignorance. Our knowledge of the past, insofar 


\section{CONCEPTS AND ASSUMPTIONS}

as it refers to concrete manifestations of the behavior of the social aggregates we call nations, states, etc., tells us clearly that the cumulative events have a pattern that can, at best, be dimly discerned only a short time ahead; and that as the period is extended, the possible errors pile up so fast that the result is useless. In dealing with a relatively short period into the future, we can actually observe some of the factors that will determine itbecause they already exist today. For example, if we wish to look 20 to 30 years ahead in population growth, we are helped by our knowledge of the population living today-since the latter will directly produce the population 20 years hence. But if we wish to know the population 2,000 years from now, there are many unborn generations whose character and composition we would have to estimate today and which we could certainly not observe during the current year. And what is true of population of human beings is true also of population of capital, whether of material or of ideas, and of any other human institutions that have a survival power some distance into the future.

This limit to our knowledge is at the bottom of our refusal to deal with projections of a long-time extension into the future. This recognition of ignorance may assume different forms. For example, one is the refusal to act upon the longer future-a refusal which unconsciously expresses a sound instinct. Since no policy is feasible, the stimulus for many a long-term projection far into the future is also absent. Another form is purely mathematical. The divergence in extrapolation of various curves, all fitted as equally good models to an observable past, compels us to limit the extrapolation to a relatively short segment beyond the current end of the observable series. Clearly, if we knew more about the future, we could discriminate among the several extrapolations. But whatever form this recognition assumes, it eliminates any infinite series of sustainable projections into the future.

Indeed, in some cases, the time extension may be reduced too much: sustainable levels may be projected too short a distance into the future for the projection to be of much value. Assume an extreme case: that on the basis of secular levels established for the current year, the projection unfolds secular levels for the next year. Obviously any addition to our knowledge, any interpretation of significance, made with such a projection lies in setting secular levels for the current year, not in their projection for the next year. For once the secular levels are established and some 


\section{CONCEPTS AND ASSUMPTIONS}

approximation to past secular trends found, little variation is possible in the quantitative magnitudes of the projection. Only extraordinarily large and variable secular movements would render such a short-span projection of longer-term changes interesting.

It follows that establishing the proper time distance of a longterm projection is a compromise between the desire not to exceed the limit of our knowledge, and the attempt to learn as much about the future as can be drawn from the past. This compromise should be made in the light of the character of the levels implied in the sustainability assumption, discussed in the preceding section; and in the light of the observation just made concerning the implicit extension of any sustainable movement beyond the date at which the levels are specified.

One further comment is relevant. If some assurance of sustainability beyond a specified date is sought, the projection must define the path leading from the present to the date in the future attached to the levels. If today we project national product to 1980 , it is not enough to calculate and present the levels in 1980. We must also show in the projection how the totals and the various components change from the secular levels of today to those of 1980. There may be different paths from a given level to a projected level, and these paths may have different meanings in terms of how much they contribute to or detract from the possibility of sustained movement after 1980. For this reason alone, even disregarding the value of such path projections for continuous checking and possible revision with the passage of time, it is desirable to trace the path from the present to whatever year the author wishes to label as the formal end of his anticipation of the future.

\section{H. USES OF PROJECTIONS}

Our discussion is necessarily in general terms. In this paper we cannot deal with the more specific problems that arise in handling the various components, or with the relations among them that are often the substance of, and the raison d'être for, many projections. These more interesting questions are treated in the specific papers that follow. Our comments, largely introductory, deal with the central questions in all long-term national product projections-which are necessarily questions of almost formal logic, inference, definition, etc. 
The general tenor of the discussion might justifiably lead some readers to conclude that the formal grounds for long-term projections of national product are tenuous indeed, and that many ambiguities remain in current formulations of such projections. Indeed, a rigorous scrutiny of the bases was bound to yield somewhat disturbing results. The rapidity and complexity of economic changes and the difficulty of objective study of human society are formidable obstacles to the formulation of tenable theories, and consequently to the establishment of elements of order in the past and the testing of generalizations against changes in relevant conditions-the two indispensable bases for valid projections. Nor, since the past is so imperfectly understood, is it surprising that there are difficulties in determining the proper limits of aggregation and disaggregation in structuring the projections, questions as to the meaning of the usually assumed stability of price levels, and ambiguities in the definition of levels projected as sustainable and of the time span to which they are supposed to apply.

The attempt to bring these problems to the surface was not made to read projections out of court. It was made rather in the hope that recognition of the problems might stimulate a more specific treatment of assumptions and contents. Such treatment is important because projections are a form of peering into the future and the latter is an indispensable part of active life.

Extrapolations into the future, whether empirically founded projections or hunches that are revealed only by their consequences in action, are being made and will continue to be made. We live in the present and cannot avoid the future; the decisions we make today will affect tomorrow. Indeed, many of them must look toward a longer-range future. Such decisions, whether made against the background of articulated forecasts or out of a subconscious but often quite strong feeling about the climate of life to be expected, imply projection in the sense of some view of the future. The choice is not between making and not making an extrapolation into the future; it is between making the projections in overt and sometimes quantitative terms, and proceeding by feel and by faith. Even inaction implies some picture of the future.

Thus the fact that it may be difficult, in the kind of examination that was carried on above, to establish the validity of the projections or to follow through fully on their implications does not 


\section{CONCEPTS AND ASSUMPTIONS}

deny their usefulness. The major use of these intellectually hazardous undertakings is naturally for policy-making. Action is directed toward the future, and the choice of action can be assisted by some specification of the future. The need for action may be motivated by some desirable goals, actively pursuedin which case the projections are often made to demonstrate that the desirable goals can be attained. Or the need for action may arise from some overriding necessity, not always pleasant or desirable-in which case the projection is made to see how the unpleasant necessity can be met without more cost or unpleasantness than need be. Or sometimes the need for action is on the part of an agent who must accommodate himself to whatever national product levels can be reasonably expected, e.g., a business enterprise wishes to have a glimpse of the national economy in the future so as to guide its actions more intelligently.

There are other types of uses, however, to which national product projections are not put, and it is of interest to list these. I know of no long-term projection that has been prepared in order to test a theory of long-term growth of the economy-the way a prediction would be used to test a theory in some of the more developed experimental sciences. National product projections have not been used in this way because there is no existing theory sufficiently articulated and empirically founded to warrant such a test. Nor are national product projections used for drawing alternative pictures of the future for choice by society-the kind of forward-looking statements that might focus public opinion upon important issues as an aid to their solution. National product projections are too detailed and technical to serve well (like utopias, and many a highly superficial but general theory) as the focus of widespread public discussion. With this country's passion for statistics, however, there has been some attempt to broadcast national product projections far and wide as expressions of economic ideals and goals. These attempts suffer from the fact that such projections, intended as elaborate quantitative pictures of feasibility, are limited to too narrow a set of assumptions. Many socially desirable ends that should be considered in any useful discussion of alternative actions for economic society cannot be translated into projections because the empirical bases for a quantitative statement are lacking.

This limitation of national product projections to uses closely geared to action may seem so obvious as not to require emphasis. 
Nor does one need to emphasize that if action is considered, it is desirable to have even a rough and approximate picture of prospects that has some empirical foundation, granted that (for reasons brought out earlier) it involves some basic assumptions that cannot be fully validated and some ambiguities and margins of errors due to an incomplete picture of the past. All this is obvious enough. But if national product projections find their justification primarily in their use for policy problems, if they are calculated despite their roughness and despite their possibly inadequate basis because they are better than proceeding by hunch and by guess, their authors and users ought to recognize this. Indeed, one might argue that presentation of any national product projections should begin with a clear statement of the types of action, of the lines of policy choice involved, that justify the effort to project despite the limitations, difficulties, and unavoidable margins of error in the result. Such a statement of uses might provide a guiding line for many decisions that must be made in formulating the projection and its various parts. It might also serve to prevent unwarranted uses of the projections, and make it clear that projections are not predictions thoroughly grounded on empirical observation or on an established theory of change of the national economy.

\section{O M M E T}

\section{Gerhard Colm, National Planning Association}

At the very end of his paper, Mr. Kuznets states that in considering policy actions, a rough and approximate picture of the prospects can be of value, as long as the limits and possible errors are recognized. This, he believes, is too obvious to require emphasis. I believe that his last paragraph, far from being obvious, could stand considerable elaboration. Most of Kuznets' paper consists of a very discouraging enumeration of all the difficulties in long-term economic projection. If, in his final paragraph, Kuznets reaches the conclusion that these projections can be of some service in considering policy action, why, then, must he be so discouraged? I believe he is discouraged because subconsciously he measures the validity of economic projections by a yardstick borrowed from "the greener pastures of the experimental scientists" ( see Section C, footnote 3), and from "some of the more developed experimental sciences" (Section H). Economic 


\section{CONCEPTS AND ASSUMPTIONS}

projections obviously do not stand up if measured with a yardstick that is not applicable. If Kuznets is skeptical about our ability to make unqualified long-range economic forecasts, I share his skepticism. I am satisfied, however, if projections are suitable for the role they were devised to play, namely, to serve as an aid in economic policy deliberations.

Kuznets is longing for a theory of economic growth which "demonstrates that long-term changes in national product invariably follow a specific pattern expressible by a given curve" (Section C). Any such theory would miss the essence of social or political economics. It is incompatible with the peculiarities of economic development, which is largely influenced by individual and collective responses to economic facts and aspirations. It is futile to search for a formula or curve by which past data can simply be extrapolated into the future. On the other hand, Kuznets is right in saying that no meaningful statement about the future is possible unless we assume that some future economic relationships and responses can be estimated on the basis of past experience. That, however, is not the same as thinking that the curve of the future gross national product in the aggregate and component parts can be found by the extrapolation of past data.

Kuznets believes that if the future is not determined by the past, "the very possibility of projections is denied." The principle of indeterminacy-that observation influences the behavior of the subject matter under observation-plays a role in some fields of physics without preventing the possibility of statements about probabilities in those fields. The same principle is of paramount importance in economics, where the behavior of individuals, groups, and governments is influenced by conscious action, which in turn is influenced by observation.

Kuznets recognizes "intentions" as one of the specifying conditions in preparing projections. The necessary introduction of this sort of assumption destroys the possibility of the mathematical projection for which he is longing. But it does not destroy the use of projections in studying the economic implications of the possible behavior of individuals, groups, and governments. For example, we may want to assess the probable economic implications and effects of an armament program, a change in tax rates, an increase in wage rates, or a change in business sentiment. The answer to such questions cannot be found by extrapolating the national product according to any curve or formula. But the study 


\section{CONCEPTS AND ASSUMPTIONS}

of policy problems of this kind can gain greatly by the use of long-range projections.

The fact that projections can be directly related to the exploration of policy measures has been mentioned in the discussion in a manner that may lead to misinterpretation. The policy objective enters the projection in the formulation of the question and in the interpretation of the answer. The preparation of the projection itself should not be influenced by any policy considerations. If the question is, for instance, what inflationary pressure is likely to result from a $\$ 50$ billion defense program, assumptions must be made as to the attitude of business and consumers. It would be wise to prepare projections under two alternatives: (1) that consumers and business believe the international situation is serious and may deteriorate even further; (2) that consumers and business believe that a relaxation of international tension is in sight. The first assumption leads of course to greater, the second to smaller, inflationary pressure. If both attitudes have a certain degree of probability, it would be wrong for the economist to overlook either of them.

In deriving recommendations for a long-range anti-inflation program, however, it may be wise and appropriate to consider only the case in which the effect of the additional defense spending is aggravated by the expectation that defense spending may have to be continued over a considerable period of time or even may have to be stepped up. Whether this is or is not the most probable assumption, it is the safest assumption for formulating a policy program. It is easier to relax anti-inflationary policy measures if and when the community anticipates an early curtailment in the defense program, than to tighten up measures in case of expectation of increased international tension. This is what I believe Arthur Smithies has called the strategic assumption of projections. It might also be called the "umbrella" principle. We take an umbrella with us not only when the weather forecast is that rain is most probable, but also when some possibility of showers is forecast. But in this case, as in economic projections, we do not want the forecast colored by a "strategic" bias. We want to decide for ourselves when we prefer the risk of getting wet to the discomfort of carrying an umbrella.

\section{Solomon Fabricant, New York University}

Among his many acute observations, Mr. Kuznets rightly points 
to the ambiguity involved in the assumption of fixed relative prices. With our attention thus drawn to the question of prices, we may go on to wonder about the significance of the assumption of constancy in the general price level.

There are two reasons for presuming that this question may be of some interest. First, there has been a good deal of speculation about, and some belief in, the influence of rising and falling general price levels on national product and on its composition and distribution. The gold and silver discoveries of the New World are said to have aided in the establishment of capitalism-that is, in helping to tilt upward the trend of national product-and to have done so by expropriation of the landed interests, lags in wages, etc. Similarly, some believe business expansions to be longer and contractions shorter-that is, growth more rapidwhen price trends are up, with and perhaps because of related effects on income distribution.

Second, when so many economists think continuing inflation is in the cards, its possibility and its consequences must be given explicit recognition in projections of the aggregate and composition of national product. Some pessimists emphasize the possibility of an ultimate "big bust," while others talk of gradual expropriation of the capitalists-but both seem to believe that the force which may have helped bring capitalism into the world will now help bury it.

Whatever one may think of the merits of these speculations about the effects of general price trends-and I confess to considerable skepticism-should they not be referred to? Anything like a full-scale analysis would, of course, become extremely involved. What effect, for example, would continuous inflation have on profits and thus on income and excess profits taxes, and thus on investment, and so on, first, in the absence of a major revision of current accounting practices, and, second, in the event that revision occurred and were widely accepted? But to admit that the question is exceedingly complex is in itself an interesting comment on the nature of long-term projections.

Mr. Kuznets has elsewhere emphasized a point that must also be remembered here: Projections for a country are projections for one member of an evolving society of nations. The impact of the world economy will, of course, be recognized in a general assumption as to war or peace and in particular assumptions about the factors determining the international investment bal- 


\section{CONCEPTS AND ASSUMPTIONS}

ance, but it needs recognition in connection with other matters also, such as change in industrial structure. For example, the decline in agriculture and increase in manufacturing in Britain and the United States were associated with important developments in other countries. Therefore projections of change in these industries assume that certain changes are taking place elsewhere. 